\title{
Family planning requirements in the adult congenital heart disease clinic
}

\author{
Helen Leonard, John J O'Sullivan, Stewart Hunter
}

\begin{abstract}
Objective-To determine whether women with congenital heart disease were receiving appropriate advice on contraception. Setting-Adult congenital heart disease clinic in a tertiary cardiac referral centre. Design-Questionnaire administered to 35 consecutive female patients attending the adult congenital clinic. The cardiologist assessed what the risk would be if each patient used an oestrogen containing contraceptive pill (OCP).

Results - Of the 33 patients admitted to the study 6 patients thought their heart condition precluded them from taking an OCP when in fact it did not and 3 incorrectly said that an OCP would be suitable for them. Three women with relatively minor lesions had been incorrectly denied the OCP and 2 further patients were using inappropriate methods. There had been 6 unwanted pregnancies in the total group. Conclusions-Many women with congenital heart disease do not know the most appropriate method of contraception for them or have received incorrect advice. It is often patients with less severe lesions who receive the most inappropriate advice. It is clear that the family planning needs of this population are currently poorly catered for. Each unit must ensure that the information necessary in making informed decisions on contraception is available to the doctor advising on family planning.
\end{abstract}

(Heart 1996;76:60-62)

Keywords: adult congenital heart disease; contraception

An increasing number of patients with congenital heart disease are surviving to adult life. Women with congenital heart disease may need specialist advice about pregnancy and contraception. There are special considerations in this group of patients; in particular, some of them may not be suitable for the combined oral contraceptive pill. The following study was carried out to ascertain whether the women seen in an adult congenital heart disease clinic were receiving appropriate advice on contraception.
Patients and methods

We surveyed 35 consecutive female patients who attended the adult congenital heart disease clinic in Freeman Hospital. This is the regional centre for congenital heart disease and most patients attending the clinic have been referred from paediatric cardiology clinics. Because patients with simple and corrected lesions are usually discharged, the patients attending this clinic tend to have residual haemodynamic problems or complex lesions.

The data were collected by questionnaire. It was made clear that it was optional and the information confidential. If the patient attended with her parents she was asked to fill the questionnaire in on her own. Women with mental impairment that prevented them from answering some of the questions were excluded from the study. A cardiologist (blinded to the results of the questionnaire) reviewed each patient's records and invasive and non-invasive data and assessed the risk of taking the combined pill and the risk of the patient undertaking a pregnancy. The oestrogen containing pill was deemed to be an inappropriate choice if it was considered that a rhythm abnormality, increased atrial size, sluggish flow in a venous conduit, poor ventricular function, pulmonary vascular disease, or hyperviscosity would increase the risk of venous or arterial clot formation.

The assessment of risk requires taking into account the individual patient's history and details of previous operations and complications. It was not possible to assign risk simply on the basis of a diagnostic label.

Results

Thirty three correctly completed questionnaires were used in the analysis. The patients ranged in age from 16 to 48 years (mean 25 years). The results of the questionnaire were divided as follows:

\section{SOURCE OF FAMILY PLANNING ADVICE}

Of the 25 women who had discussed contraception with a doctor, seven had discussed it with a cardiologist and 17 had approached their general practitioner-in three of these cases the general practitioner had sought further advice from the hospital cardiology clinic. Four had been to a family planning clinic and two had discussed it with a health visitor. Some women had discussed it with more than one person. \section{Department of Paediatric Cardiology, Freeman Hospital, Newcastle upo Tyne NE7 7DN. \\ Accepted for publication 20 March 1996 H Leonard $S$ Hunter}


In only 12 cases had the subject of contraception been brought up in advance by the doctor. Similarly, only 17 of the patients had been counselled in advance of becoming pregnant on the implications of a pregnancy.

PATIENTS KNOWLEDGE OF THE INFLUENCE OF THE CARDIAC DIAGNOSIS ON CONTRACEPTIVE CHOICE

Twelve patients were unsure whether their heart condition would have an impact on their method of contraception. (It is possible that completing the questionnaire raised doubts in women whose heart disease was insignificant.) In most (11) their cardiac condition was not a contraindication to the combined pill. Contraception used in this group of 12 was the combined pill (4), tubal ligation (3), condoms (1), and partner's vasectomy (1) and three were not sexually active.

Twelve women correctly knew how their cardiac diagnosis influenced their contraceptive choice.

The remaining nine were incorrect. In six this was because they felt their heart condition was relevant to contraceptive choice when it was, in fact, insignificant. However, three women who had heart disease that was relevant to the choice of contraception were not aware of this.

\section{CONTRACEPTIVE METHOD USED}

Six patients were not sexually active. In two patients their present contraceptive method was inadequate and more suitable methods easily available. The oestrogen containing pill had been inappropriately prescribed to a patient with mitral valve prolapse, moderate mitral regurgitation, and an enlarged left atrium. Three patients had been unnecessarily denied the oral contraceptive pill: one with mild to moderate pulmonary stenosis, one with a small residual ventricular septal defect, and one with Marfan syndrome and mild aortic regurgitation. This last patient had used condoms after being refused the pill and had had two unplanned pregnancies.

\section{CONTRACEPTIVE FAILURES}

There had been six unplanned pregnancies in two patients. One of these had Marfan syndrome and has already been discussed. The other had had surgical correction of tetralogy of Fallot and a permanent endocardial pacemaker and had never received family planning advice after being refused the pill by her general practitioner. She had had two terminations and eventually a tubal ligation.

\section{WHERE PATIENTS WOULD LIKE TO RECEIVE}

\section{FAMILY PLANNING ADVICE}

Most patients $(25,75 \%)$ preferred to get advice on contraception from their general practitioner, four (12\%) preferred a family planning clinic. Only four said they would prefer the hospital clinic to advise them on contraception. Two of these patients had previously been given incorrect advice by their GPs and a further patient had severe and complex congenital heart disease.

\section{Discussion}

The population of adults with congenital heart disease is increasing and it is also diverse in terms of complexity of diagnosis and haemodynamic status. There is little published information about contraception in these patients and some texts incorrectly regard all adults with congenital heart disease as a homogeneous group. ${ }^{1}$

The risk of clot formation and pregnancy often cannot be predicted from the cardiac diagnostic label alone and the individual patient's history needs to be reviewed. The combined oral contraceptive pill is not suitable for women who have a structural, functional, or haematological abnormality that increases their risk of thromboembolism. ${ }^{2}$ Some of these patients can be easily identified, but inappropriate prescription of the oestrogen containing pill was evident in our study group although it was not a common problem. Many patients have no cardiac contraindication to taking a low oestrogen pill. In our study several of these patients had been denied the combined pill unnecessarily, and even when condoms are used, this can result in unplanned pregnancy. ${ }^{3}$

This failure to identify women for whom the pill may or may not be suitable needs to be addressed. It is not clear where family planning issues for these patients are best discussed. It was apparent from our study that most patients would prefer to discuss contraception with their general practitioner or local family planning clinic. A general practitioner or family planning adviser cannot be expected to have a detailed knowledge of every patient's cardiac diagnosis or haemodynamic status or its implications

A busy hospital outpatient clinic is rarely the best place for a young woman to discuss contraception. She is confronted with a variety of medical staff and may have attended with parents. A cardiology consultant used to dealing with children may be unaware of their patients' transition into sexually mature individuals, often at comparatively young ages. Similarly cardiologists cannot be expected to be experts in contraception.

There are several situations-for example, patients taking oral anticoagulants-where some controversy exists about the safety or otherwise of oestrogen containing pills ${ }^{4-7}$ and a combined approach between the family planning experts and congenital heart disease specialist is needed.

The family planning needs of adult patients with congenital heart disease are presently poorly provided for. Simply denying patients the pill is not adequate advice and the service must use a team approach to cater for the needs of patients with complex problems. Perhaps these issues should be addressed routinely by the paediatric cardiologist when these patients are being discharged from the paediatric clinic. Each individual unit should decide on the best way to provide this service to its patients.

We thank Dr Vivienne O'Sullivan of the family planning service for her expert advice on family planning issues. 
1 Fraser IS. Contraceptive choices for women with "risk factors". Drug Safety 1993;8:271-9.

2 Vessey M, Mant D, Smith A. Oral contraceptives and venous thromboembolism. BrMed F 1986;292:526.

3 Abdalla $M$, Mostafa E. Contraception after heart surgery. Contraception 1992;45:73-80.

4 Bodgers J, Huikeshoven F, Lotgering F. Complications of anticoagulant therapy in ovulatory women. Lancet 1991; anticoagulant

5 Comp PC, Zacur HA. Contraceptive choices in women with coagulation disorders. Am f Obstet Gynecol 1993; 168:1990-3.

6 Turpie A, Gent M, Laupacis A, Latour Y, Gunstensen J, Basile F, et al. A comparison of aspirin with placebo in patients treated with warfarin after heart valve replacement. N Engl F Med 1993;329:524-9.

7 Fihn SD, McDonell M, Martin D, Henikoff J, Fihn SD, McDonell M, Martin D, Henikoff J,
Vermes D, Kent D, et al. Risk factors for complications of chronic anticoagulation. Ann Intern Med 1993;118: of chron.

\section{ABSTRACTS IN CARDIOLOGY}

\section{Atherosclerosis of the aortic arch and the risk of ischaemic stroke}

Atherosclerotic disease of the thoracic aorta is a potential source of cerebral embolism. Amarenco et al found a strong independent association between atherosclerotic disease of the aortic arch and the risk of ischaemic stroke. Few reports have focused on the ascending thoracic aorta as a potential source of embolic strokes and such evidence is needed before the thoracic aorta is included in the routine transoesophageal (TOE) assessment of heart patients with embolic stroke. TOE could in some cases alert surgeons to be vigilant during aortic clamping to avoid perioperative thromboembolism. This advantage may be more relevant to coronary angiography, however, as it might be better to use the right brachial artery cut-down technique (Sones) rather than the femoral artery route (Judkin) to reduce the risk of the catheter dislodging plaque in patients shown by TOE to be at high risk before catheterisation.

The retrospective detection of thick plaques by TOE unfortunately does not contribute to patient care and if it has no influence on management it is questionable whether the mild discomfort and cost of TOE in all patients with embolic stroke can be justified. It is more reasonable to regard atherosclerotic plaques detected by TOE or by any other means simply as evidence of diffuse atheromatosis and, as with coronary or peripheral vascular disease, to treat patients with antiplatelet agents, especially if they are over the age of 60 .

PETROS NIHOYANNOPOULOS

\section{Atherosclerotic disease of the aortic arch and the risk of ischemic stroke}

Pierre Amarenco, Ariel Cohen, Christophe Tzourio, Bernard Bertrand, Marc Hommel, Gérard Besson, Christophe Chauvel, Pierre-fean Touboul, Marie-Germaine Bousser

\section{Abstract}

Bachground-Atherosclerotic disease of the aortic arch has been suspected to be a potential source of cerebral emboli. We conducted a study to quantify the risk of ischemic stroke associated with atherosclerotic disease of the aortic arch.

Methods-Using transesophageal echocardiography, we performed a prospective case-control study of the frequency and thickness of atherosclerotic plaques in the ascending aorta and proximal arch in 250 consecutive patients admitted to the hospital with ischemic stroke and 250 consecutive controls, all over the age of 60 years.

Results-Atherosclerotic plaques $\geqslant 4 \mathrm{~mm}$ in thickness were found in 14.4 percent of the patients but in only 2 percent of the controls. After adjustment for atherosclerotic risk factors, the odds ratio for ischemic stroke among patients with such plaques was $9 \cdot 1$ (95 percent confidence interval, 3.3 to $25.2 ; \quad P<0.001)$. Among the 78 patients who had brain infarcts with no obvious cause, $28 \cdot 2$ percent had plaques $\geqslant 4 \mathrm{~mm}$ in thickness, as compared with $8 \cdot 1$ percent of the 172 patients who had infarcts whose possible or likely causes were known (odds ratio, 4.7; 95 percent confidence interval, 2.2 to $10.1 ; P<0.001$ ). Plaques of $\geqslant 4 \mathrm{~mm}$ in the aortic arch were not associated with the presence of atrial fibrillation or stenosis of the extracranial internal carotid artery. In contrast, plaques that were 1 to $3.9 \mathrm{~mm}$ thick were frequently associated with carotid stenosis of $\geqslant 70$ percent.

Conclusions-These results indicate a strong, independent association between atherosclerotic disease of the aortic arch and the risk of ischemic stroke. The association was particularly strong with thick plaques. Atherosclerotic disease of the aortic arch should be regarded as a risk factor for ischemic stroke and as a possible source of cerebral emboli. (N Engl Y Med 1994;331:1474-9). 